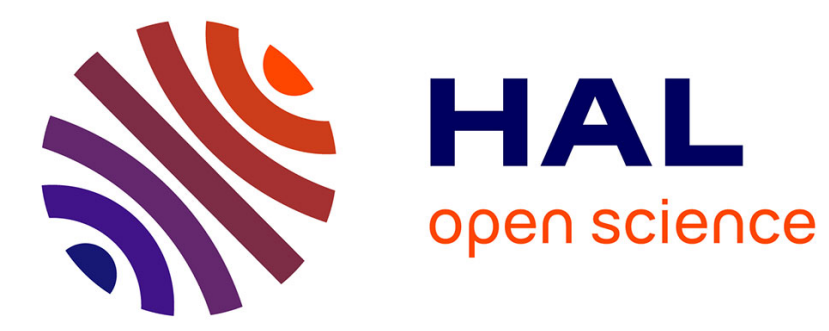

\title{
Deux types d'imparfait atténuatif
}

Lotfi Abouda

\section{To cite this version:}

Lotfi Abouda. Deux types d'imparfait atténuatif. Langue française, 2004, Procédés de modalisation: l'atténuation, 142 (1), pp.58-74. halshs-01471276

\section{HAL Id: halshs-01471276 \\ https://shs.hal.science/halshs-01471276}

Submitted on 19 Feb 2017

HAL is a multi-disciplinary open access archive for the deposit and dissemination of scientific research documents, whether they are published or not. The documents may come from teaching and research institutions in France or abroad, or from public or private research centers.
L'archive ouverte pluridisciplinaire HAL, est destinée au dépôt et à la diffusion de documents scientifiques de niveau recherche, publiés ou non, émanant des établissements d'enseignement et de recherche français ou étrangers, des laboratoires publics ou privés. 


\section{Lotfi ABOUDA}

CORAL

Université d'Orléans

\section{Deux types d'imparfait atténuatif}

\section{INTRODUCTION}

La plupart des travaux antérieurs traitant de l'imparfait dit d'atténuation sont des travaux généralistes, dans lesquels cet emploi - considéré, par une sorte de hiérarchisation souvent implicite, comme un emploi atypique, voire marginal - est étudié "en bout de course " ', et dans le cadre d'analyses pour le moins sommaires. Cet état de choses n'est contredit que par un article de Berthonneau et Kleiber (1994), dans lequel ils proposent un traitement global et détaillé de ce qu'ils appellent l'imparfait de politesse, qui englobe, selon eux, aussi bien l'IMP forain, illustré par:

(1) (a) Qu'est-ce qu'elle voulait la petite dame?

que l'lMP d'atténuation, qu'ils illustrent par :

(1) (b) Je voulais/venais vous demander un petit service.

Leur traitement admet que, même dans ces emplois, il existe toujours une situation passée à laquelle renvoie en partie l'IMP, considéré comme temps anaphorique méronomique. Si ce traitement - chronologique - nous semble assez convaincant pour l'IMP forain, et pourrait même s'appliquer à certains énoncés atténuatifs, son extension à tous les énoncés à imparfait d'atténuation ne nous semble pas légitime ${ }^{2}$. C'est que la notion d'imparfait d'atténuation, telle qu'elle est retenue habituellement, n'est pas homogène, et semble en fait correspondre à deux types d'IMP, qui s'opposent sur les plans syntaxique, chronologique, et discursif.

Le premier - que nous appellerons imparfait de justification - sert, sur le plan discursif, à justifier une situation présente, dont le locuteur pense qu'elle pourrait déranger l'interlocuteur (le plus souvent, une intrusion physique ou téléphonique), et dont il présente les causes sous forme d'intentions passées. Syntaxiquement, il se

1. Berthonneau \& Kleiber (1994: 64).

2. Une réserve analogue est exprimée par Touratier (1996: 1.38-140)). 
rencontre dans des contextes assez divers, et avec une liste relativement ouverte de verbes, même si on peut voir dans venir son représentant typique :

(a) Je venais passer l'aspirateur.

Le second, qui sera baptisé imparfait de déperformativité, loin de localiser le procès dans un passé accessible à l'interlocuteur, sert tout simplement à "désamorcer" un acte de langage actuel, le plus souvent de type assertif ou interrogatif. Sur le plan syntaxique, il se rencontre quasi exclusivement avec le verbe vouloir gouvernant une infinitive en dire/demander:

(2) (b) Je voulais te dire aussi que je suis inscrite au parti communiste...

L'opposition entre IMP déperformatif et IMP justificatif peut être décrite, en première approximation, de la manière - quelque peu lapidaire - suivante : si $J e$ venais passer l'aspirateur est bien l'atténuation de Je viens passer l'aspirateur, Je voulais vous dire que je suis inscrite au PC se présente comme l'atténuation de Je vous dis que je suis inscrite au PC, et non pas de Je veux vous dire que je suis inscrite au PC. On comprend ainsi pourquoi nous parlons de déperformativité : l'IMP de vouloir sert à désamorcer l'acte de langage - en l'occurrence assertif - qui aurait été accompli par le verbe performatif dire dans l'énoncé non atténuatif correspondant, i.e. Je vous dis que je suis inscrite au PC.

Cette distinction ne signifie pas que les deux types d'emplois soient forcément contradictoires - certains énoncés sont passibles des deux analyses -, pas plus qu'elle ne les empêche d'avoir un certain nombre de propriétés communes, qui justifient qu'on les regroupe au sein de la classe d'lMP d'atténuation, et notamment les deux traits définitoires suivants :

(i) le renvoi à une situation (encore) valide au moment de l'ënonciation : ce trait sera considéré comme pertinent toutes les fois que l'IMP peut être remplacé par un présent et que la substitution produit, parallèlement ou non à un changement chronologique, un écart " stylistique " entre un énoncé " poli » et un énoncé non marqué ;

(ii) l'expression d'un effet "stylistique " qui relève de l'atténuation, une des déclinaisons possibles de la modalisation, conçue ici comme une modification dans la prise en charge par le locuteur d'un énoncé théoriquement neutre. Plus précisément, l'effet atténuatif consiste, ainsi que le rappelle P. Haillet (2002 et ici même), à présenter un énoncé " comme moins direct (moins catégorique, moins abrupt, moins péremptoire) " que l'énoncé non marqué correspondant.

La traduction exacte de ce dernier trait, qui n'a pas été enregistré de la même manière chez tous les observateurs, permettra finalement d'opposer les deux types d'énoncés " atténués", et partant les deux types d'IMP. Ce sera l'objectif ultime de cet article, qui s'attachera, pour ce faire, à détailler le fonctionnement de l'IMP atténuatif, en décrivant les conditions syntaxiques et/ou discursives qui déterminent ou favorisent son apparition. Les questions syntaxiques que nous nous poserons sont multiples. Elles concernent notamment les types de structures syntaxiques dans lesquelles on peut rencontrer ces emplois, les types de verbes à l'IMP, les personnes grammaticales, les contraintes sur les éventuels verbes gouvernés, etc.

Or, une étude syntaxique de l'IMP nécessite l'examen d'un nombre conséquent de données, que nous n'avons pas pu trouver dans les travaux antérieurs qui fournissent généralement un tout petit nombre d'exemples attestés, souvent les mêmes, ou se contentent d'exemples fabriqués. Aussi, la première phase de cette 
recherche a consisté à collecter des données nouvelles, dans différentes bases de données informatisées, aussi bien écrites (notamment FRANTEXT, où il s'est agi de relever les occurrences de chacune des formes verbales considérées, dans différents genres textuels, à compter de 1960), qu'orales (notamment le corpus d'Orléans ${ }^{3}$ ).

Les résultats de ces collectes, même s'ils sont riches en enseignements, méritent d'emblée d'être relativisés. D'abord parce que le type d'IMP recherché ne peut se rencontrer que dans des contextes pragmatiques très étroits, et donc finalement peu présents dans les corpus examinés. Ceci est particulièrement vrai des corpus oraux qui, correspondant le plus souvent à des entretiens, n'enregistrent quasiment jamais les micro-situations pragmatiques où il est théoriquement possible de rencontrer un IMP d'atténuation. Ensuite, parce que les requêtes formulées ne peuvent concerner, pour diverses raisons, qu'un certain nombre de formes verbales, à savoir : aimer, appeler, avoir, désirer, préférer, souhaiter, téléphoner, venir et vouloir, conjuguées à l'IMP, et à la première personne du singulier. Ce sont là les principales formes verbales dont l'examen nous a paru particulièrement utile, soit parce qu'elles pouvaient relever de l'IMP d'atténuation, soit parce qu'elles entretiennent des rapports sémantiques évidents avec les premières. Il va de soi que ces requêtes dépendent étroitement et de l'intuition du chercheur, et des principales hypothèses habituellement proposées...

\section{LES VERBES AUTORISANT L'ATTÉNUATION}

N'importe quel verbe à l'IMP ne permet pas la production d'un effet d'atténuation. La question se pose donc de savoir quels sont les verbes qui permettent cet effet de sens. Même s'il n'y a pas consensus sur le sujet, il ressort des exemples habituellement fournis et des commentaires descriptifs disponibles que le verbe à l'IMP doit soit correspondre au verbe opérateur venir, soit appartenir à la classe des verbes désidératifs, représentée quasi systématiquement par vouloir.

Il est crucial de vérifier ces affirmations par l'examen de corpus, en essayant notamment de répondre à la question de savoir si la lecture atténuative de l'IMP est disponible avec des verbes désidératifs autres que vouloir, et si, plus généralement, elle est possible avec des verbes autres que les verbes désidératifs et venir. Nous examinerons donc successivement vouloir, venir et les éventuels autres verbes autorisant une atténuation à l'IMP, en cherchant à expliciter leurs propriétés syntaxiques saillantes.

\section{I.I. Vouloir}

D'après les descriptions antérieures, l'exemple type de l'énoncé atténuatif est

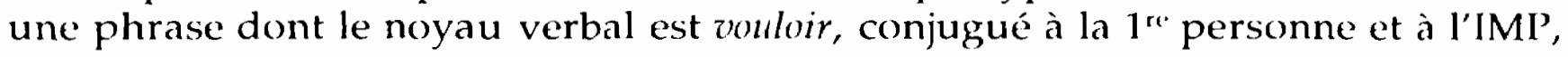

\footnotetext{
3. Ce corpus est l'un des plus importants corpus du français oral actuellement disponibles. Il est actuellement en cours de transcription au département de linguistique de la K.U. I.cuven, et la partie disponible (qui correspond à \pm 80 heures d'enregistrement) ést déjà en consultation libre sur le site http://www.bach.arts.kuleuven.ac.be/elicop/.
} 
et dominant une subordonnée infinitive en dire/demander, comme dans les énoncés attestés suivants:

(3) (a) - Je viens acheter un bijou, dit Paulo, pour ma fiancée. Une bague. Mais avant je voulais te dire qu'Adèle est très malheureuse et qu' elle ne vit que dans l'espoir de te retrouver.

(b) - Je voulais savoir si la supérieure a parfois l'occasion de lire des livres français.

- Oh non! Je suis trop occupée!

La plupart des traitements - considérant l'imparfait comme un temps du passé expliquent l'effet atténuatif par la conversion de l'écart chronologique en écart stylistique, les propriétés aspectuelles de l'IMP se chargeant d'assurer la continuité du procès dans l'actualité. Le mécanisme de cette conversion est bien expliqué par M. Wilmet (1996: 205):

imparfait dit "de politesse », « d'atténuation ", "illocutoire " ou « à sens de présent ", mais, en fait, un vrai passé : la courtoisie consiste précisément à enfouir, à reléguer l'intention de "demander un petit service", en laissant l'interlocuteur fictivement libre de percevoir ou non l'actualité de la requête (= “ je voulais, mais si cela vous gêne, je ne veux plus ») [...].

Si cette explication nous semble adéquate pour rendre compte des énoncés atténuatifs de type justificatif, dont fait sans doute partie le fameux Je voulais vous demander un petit service, elle nous paraît beaucoup plus discutable quand il s'agit de rendre compte des énoncés typiques comme (3). Non seulement nous ne voyons pas à quelle situation passée accrocher - naturellement - le procès à l'IMP, mais en plus, l'explication de type “ je voulais, mais si cela vous gêne, je ne veux plus " nous paraît tout simplement contre-intuitive face à des énoncés comme (3) qui se présentent comme un mélange de politesse et de fait accompli : quelle que soit l'attitude de l'interlocuteur, une fois que $\mathrm{l}^{\prime}$ on a dit le voulais dire (de'mander) que (si) P, c'est dit... et même fait, et il n'y a plus aucun moyen d'y renoncer !

Pour nous, cet emploi doit être nettement distingué de l'emploi justificatif. Ici, l'effet atténuatif correspond tout simplement à la neutralisation de l'acte du langage direct qui aurait été accompli par l'énoncé non atténuatif correspondant. Autrement dit, l'atténuation correspond ici à un déguisement d'un acte de langage (trop) direct. Nous y reviendrons.

\section{I.I.I. Vouloir: représentant des verbes désidératifs ?}

La majorité écrasante des exemples habituellement fournis pour illustrer l'IMP d'atténuation ont pour noyau verbal vouloir. S'agit-il là d'une propriété exclusive de ce verbe, ou existe-t-il d'autres verbes, sémantiquement proches, susceptibles d'autoriser le même effet de sens?

La plupart des travaux consultés se montrent à ce propos laconiques, soit en parlant simplement de "quelques verbes " sans dire lesquels, soit en se contentant de fournir des exemples avec vouloir, mais en évitant de se poser explicitement la question, soit encore en parlant de verbes désidératifs sans les définir, qu'on illustre alors quasi-systématiquement par vouloir, et, quelquefois, par souhaiter et désirer, mais exclusivement au sein d'exemples fabriqués.

Une vérification sur corpus nous ayant semblé utile, nous avons décidé de relever l'ensemble des énoncés ayant pour noyau un verbe désidératif ou un verbe 
sémantiquement proche, à savoir vouloir, désirer, souhaiter, aimer, et préférer, et de vérifier s'ils correspondent ou non à des énoncés atténuatifs.

Les résultats obtenus sont sans appel : des cinq verbes considérés, seul vouloir à l'imparfait et à la $1^{\text {re }}$ personne du singulier se rencontre dans des énoncés atténuatifs, aussi bien dans Frantext (43 énoncés atténuatifs sur un total de 1222 énoncés avec je voulais) que dans le corpus d'Orléans (3 énoncés atténuatifs sur un total de 57).

Ces résultats confirment en partie les descriptions antérieures qui excluent de la classe des verbes pouvant produire une atténuation à l'IMP aimais et préférais : ces verbes ne sont jamais atténuatifs, parce que, situant clairement le procès dans le passé, ils ne remplissent pas la première condition pour ce type d'imparfait, à savoir le renvoi à une situation (encore) valide au présent.

En revanche, en ce qui concerne la sous-classe des "verbes de volonté " (Willems, 1981), qui regroupe vouloir, souhaiter et désirer, les données quantitatives obtenues semblent contredire certaines descriptions antérieures, qui ne voient dans vouloir qu'un simple représentant des verbes autorisant l'expression d'une atténuation. Il nous est en effet difficile d'attribuer à souhaiter et désirer la même faculté que vouloir de rendre un effet atténuatif à l'IMP, puisque aucun énoncé atténuatif avec ces verbes n'a été répertorié dans nos corpus.

Même s'il est peu probable que ces résultats constituent un accident, une vérification supplémentaire s'impose. Nous avons donc fabriqué un certain nombre de phrases avec vouloir, désirer et souhaiter et nous les avons soumises au jugement d'acceptabilité d'un certain nombre de locuteurs. Même mitigés, les résultats obtenus confirment que vouloir est de loin le mieux placé pour rendre naturellement un effet de sens atténuatif. Pour souhaiter et désirer, en revanche, les locuteurs interrogés sont partagés, et la plupart d'entre eux doutent de leurs propres jugements.

En bref, même s'il est difficile de nous appuyer totalement sur cette enquête, il se dégage tout de même une tendance, assez significative, qui revient à considérer vouloir à l'IMP comme la forme la plus naturelle et la plus commune pour l'expression d'une atténuation.

Il nous restera alors à expliquer pourquoi tous les verbes dits de volonté ne permettent pas - ou ne permettent pas aussi facilement que vouloir - la production d'un effet de sens atténuatif. Nous y répondrons en 2.3.

\subsubsection{Structures syntaxiques de vouloir à l'imparfait d'atténuation}

À la fois dans les exemples généralement fournis pour illustrer l'IMP d'atténuation et dans les commentaires descriptifs, lorsqu'ils existent, il ressort que ce type d'IMP se rencontre très généralement dans une structure syntaxique de type:

(4) Verbe à l'IMP + subordonnée infinitive

Est-ce à dire que l'IMP atténuatif de vouloir ne peut se rencontrer que dans des structures de type (4) ? La plupart des travaux antérieurs observent à ce propos la plus grande prudence, soit en se contentant de fournir des exemples qui obéissent au schéma syntaxique (4), mais sans se poser explicitement la question, soit en préférant, à l'image de Berthonneau et Kleiber (1994), présenter cette structure comme celle de "l'exemple type". S'agissant pourtant d'une question cruciale, dont dépendent en partie nos hypothèses explicatives, nous chercherons à déterminer ici avec précision les différentes structures de l'IMP d'atténuation, en répondant notamment à la question de savoir si vouloir peut gouverner, à la place de l'infinitive, un syntagme nominal (SN) et/ou une complétive. 


\section{I.I.2.I. Le constituant gouverné par l'imparfait est un SN}

Dans nos corpus, aucun exemple où l'IMP gouverne un $\mathrm{SN}$ ne relève de l'atténuation. Se pose, plus généralement, la question de savoir s'il est possible d'avoir un IMP atténuatif gouvernant un SN. Pour y répondre, nous avons entrepris de fabriquer des exemples de ce type et de les soumettre au jugement d'un certain nombre de locuteurs. À quelques exceptions près, la plupart des locuteurs interrogés ne considèrent pas des exemples comme les suivants :

(5) (a) Je voulais un maillot de bain rouge

(b) Je voulais une baguette

comme pouvant naturellement exprimer une atténuation. Ils excluent en effet à juste titre que l'on puisse ouvrir un dialogue avec des énoncés comme (5), et s'accordent à dire que ce type d'énoncés n'est naturel que s'il y a une situation passée à laquelle renvoie l'IMP. En bref, en dehors de contextes pragmatiques particuliers, et contrairement aux mêmes énoncés au conditionnel, la lecture la plus naturelle et la plus immédiate de ces exemples est temporelle.

Toutefois, il semble qu'avec certains $S N$, un certain effet atténuatif peut plus facilement être exprimé, comme c'est le cas, dans des contextes pragmatiques particuliers, des énoncés suivants, qui sont également passibles d'une lecture temporelle:

(6) (a) Je voulais juste un rendez-vous.

(b) Je voulais un petit renseignement.

Parallèlement à l'adverbe juste et à l'adjectif petit, l'IMP joue un rôle important dans l'émergence de l'effet atténuatif. Cet effet correspond-il au désamorçage d'un acte de langage (et lequel ?), ou au contraire, à la justification d'une situation présente par une intention passée?

Même dans le cas où nous analyserions ces énoncés comme des ellipses respectives de:

(6') (a) Je voulais juste vous demander un rendez-vous.

(b) Je voulais vous demander un petit renseignement.

il nous semble que c'est l'hypothèse d'un IMP justificatif qui vient plus naturellement à l'esprit. Le locuteur y explique/justifie son intrusion dans l'espace de l'interlocuteur.

En bref, la structure vouloir à l'IMP $+S N$ - marginale sur le plan quantitatif, et sur le plan interprétatif plutôt proche de l'emploi justificatif - ne sera pas considérée ici comme une structure naturelle de l'IMP d'atténuation avec vouloir.

\subsubsection{Le constituant gouverné par l'imparfait est une complétive}

La subordonnée infinitive gouvernée par l'IMP peut-elle éventuellement céder la place à une complétive ou est-elle, au contraire, absolument requise ? Cette question revient à se demander si, dans un énoncé atténuatif, le sujet de la principale (correspondant le plus souvent au locuteur) et celui de la subordonnée peuvent ne pas être co-référentiels.

Aucun des 78 énoncés de notre corpus Frantext où Je voulais domine une complétive n'étant atténuatif, nous avons entrepris, là aussi, de fabriquer un certain nombre d'énoncés de ce type, en nous interrogeant sur leurs sens dans différents contextes. Si la plupart des énoncés fabriqués ne peuvent avoir qu'une lecture temporelle, certains sont parallèlement passibles d'une lecture atténuative. Tel est, nous semble-t-il, le cas 
des énoncés suivants, qui, selon nous, ne posent pas de problème d'acceptabilité même s'ils seraient sans doute plus naturels au conditionnel :

(7) (a) Je voulais que vous nous présentiez votre projet.

(b) Je voulais que vous nous racontiez cette histoire.

Précisons simplement que la lecture atténuative, qui n'est ni la plus immédiate ni la plus naturelle, est en plus conditionnée par l'expression d'une demande. Les énoncés ci-dessus peuvent alors être facilement paraphrasés, à l'effet d'atténuation près, par les énoncés suivants à l'impératif :

$\left(7^{\prime}\right) \quad$ (a) Présentez-nous votre projet.

(b) Racontez-nous cette histoire.

Cette paraphrase nous conduit à parler ici de la neutralisation d'un acte de langage injonctif. Nous y reviendrons.

\subsection{Venir}

D'après les descriptions antérieures, vonir, conjugué à la $1^{\text {re }}$ personne et à l'IMP, et dominant une subordonnée infinitive directe, permet l'expression d'un effet atténuatif, comme dans l'énoncé (8):

(8) Je venais te dire bonsoir. (A. Gide)

Mais, contrairement à ces descriptions, qui assimilent les énoncés (3) et (8), nous pensons que l'effet de sens dont il est ici question se distingue nettement de celui produit par l'IMP de vouloir. En effet, si l'atténuation du premier type correspond à la neutralisation d'un acte de langage, elle prend dans (8) clairement la forme d'une justification de l'intrusion du locuteur dans l'univers de I'interlocuteur. En effet, à moins de s'attacher à une conception archaïque des relations entre locuteurs, il nous est difficile de concevoir la politesse comme l'atténuation d'une salutation, qui constitue elle-même un acte poli. Les exemples que nous avons pu relever dans nos corpus confirment notre intuition. L'énoncé suivant :

- Néanmoins, je suis inquiète, maître. Cet enfant parle de la mort en termes qui ne sont pas de son âge. Je venais vous demander, humblement, pour l'amour de ce disciple que vous chérissez, de vous souvenir de son âge, dans votre œuvre d'édification.

ne nous semble atténuatif que dans la mesure où il permet d'expliquer les raisons de la venue du locuteur en les présentant comme des intentions passées, ou, plutôt, des intentions dans le passé.

Cette analyse semble se confirmer par le fait que venir et vouloir ne sont pas interchangeables. Plus précisément, si certains contextes pragmatiques permettent indifféremment l'emploi de l'un ou de l'autre pour produire une atténuation, le plus souvent, venir ne peut pas se substituer à vouloir, alors que l'inverse semble toujours possible, dès lors que venir domine une subordonnée directe ${ }^{4}$. Une première explication pragmatique de cette impossibilité de substitution semble évidente : le locuteur ne peut employer lo venais + infinitif que s'il a effectué lui-même le déplacement: venir, à défaut d'exprimer toujours un déplacement spatial, le symbolise systématiquement. Mais ce n'est pas là une condition suffisante. Avec venais, le locuteur livre

4. I'récision rendue nécessaire par des énoncés comme (15(a)-(b)), où cette substitution est exclue. 
la raison principale de sa venue. Toutes les fois qu'il cherche à présenter son dire comme secondaire ou accidentel, c'est l'emploi de voulais qui s'impose :

(10) Pendant qu'il remplissait les papiers, j'ai toussé délicatement dans mon poing :

- Ah, docteur, je voulais vous dire... Il y a aussi une petite chose qui me tracasse...

où, grâce à certaines marques linguistiques, le locuteur minimise l'objet de son dire, et le présente comme marginal... tellement marginal qu'il a failli l'oublier.

Avec venir, au contraire, le locuteur met en avant l'objet de sa visite. C'est que le rôle du verbe venir est d'annoncer la raison de la venue, celui de l'IMP est de l'atténuer en la présentant au stade de la simple intention. C'est sans doute pour cela aussi que si les énoncés avec venir ne correspondent pas systématiquement à une forme de "première adresse", ils figurent très souvent parmi les tout premiers échanges entre interlocuteurs, ce qui n'est pas nécessairement le cas, ainsi que l'ont déjà observé Berthonneau \& Kleiber (1994), des énoncés avec vouloir.

Notons enfin que nous ne lions pas l'effet atténuatif de venais à la présence dans l'infinitive d'un verbe de type dire/demander. Un énoncé comme (11) produit bien un effet d'atténuation, et est en tout point comparable aux énoncés (8) et (9) :

Je venais voir si vous avez réfléchi. (Romains)

\section{I.3. Autres verbes, autres structures}

Peut-on rencontrer un IMP atténuatif avec des verbes autres que vouloir et venir? Cette question ne pouvant pas recevoir de réponse exhaustive, nous nous sommes contenté d'examiner ici deux verbes quelquefois associés à cet effet de sens, à savoir appeler et téléphonér. Si nos corpus ne contiennent aucun énoncé atténuatif avec télćphonais, nous pouvons, en guise d'illustration, emprunter ici l'exemple atténuatif suivant à Berthonneau \& Kleiber (1994) :

(12) Je vous téléphonais pour savoir si ma voiture était réparée. [au téléphonel Concernant appeler, nous avons relevé dans Frantext les deux exemples suivants:

(a) - L'Arquebuse! C'est moi ! hurlai-je dans l'appareil. Je t'appelais pour que tu me dises que tu m'aimes.

(b) ... désolé de t'avoir réveillée... Je te rappellerai demain...

- Mais non! De toute façon, je ne dormais pas encore, je venais de finir mon devoir de math.

- Justement, je t'appelais à ce sujet... Tu sais, les systèmes d'équations au premier degré?

Ces énoncés nous semblent assez proches de l'exemple suivant, relevé par P. Haillet :

(14) Nous cherchions à joindre M. Ben Laden. là l'interphone, JT de $20 \mathrm{~h}$, France 2]

et d'un certain nombre d'énoncés non typiques avec venir dominant, au lieu d'une infinitive, un syntagme prépositionnel en à ou en pour :

(15) (a) Bonsoir! Je venais pour le bureau B215... [s'adressant aux occupants d'un bureau voisin]

(b) ISABELLE. - Allô ?

ANNE. - C'est toi ?

ISABELLE. - Oui, c'est moi. Je venais aux nouvelles. Et puis j'en ai une à te donner aussi. 
Ces exemples sont comparables à l'énoncé attesté suivant avec passer - ce qui suggère que venir ne serait finalement dans cet emploi qu'un simple représentant de certains verbes de déplacement :

(16) Je passais juste pour prendre mon agenda. len entrant dans un bureau]

Dans tous ces énoncés, qui ne correspondent pas au schéma syntaxique-type habituellement reconnu pour l'emploi atténuatif, l'lMP peut être remplacé par un présent, puisque son rôle n'est pas de situer le procès dans le passé, mais de produire un effet d'atténuation qui prend en l'occurrence la forme d'une motivation polie de l'intrusion du locuteur dans la sphère de l'interlocuteur (le coup de téléphone, la sonnerie de l'interphone, ou la visite...). Il "garde » donc ainsi une certaine épaisseur temporelle, puisqu'il semble renvoyer à un moment du passé où les raisons de l'intrusion du locuteur étaient au stade de simples intentions.

\section{AUTRES PROPRIÉTÉS SYNTACTICO-SÉMANTIQUES}

\section{I. La personne grammaticale du verbe à l'imparfait}

Si, comme l'observent la plupart des travaux antérieurs, l'IMP atténuatif exige normalement d'être employé à la $1^{\text {re }}$ personne, la question se pose de savoir si cette personne, qualifiée de "normale", peut éventuellement céder la place à une autre personne.

En ce qui concerne la $2^{\prime \prime}$ personne, l'écrasante majorité des exemples dans le corpus Frantext n'ont clairement pas de sens atténuatif. Un tout petit nombre d'énoncés nous semblent toutefois devoir être examinés de plus près, dont les deux suivants :

(a) L'instrument étant en dérangement, on devait s'adresser à l'intérieur. Olivier entra d'autorité :

- B'jour, m'sieurs dames, alors, elle marche pas, la balance?

- Tu voulais te peser? demanda la pharmacienne, une dame à cheveux blancs.

- Oui, m'dame, et mon copain aussi.

(b) - à propos, dit-il, tu voulais me demander un conseil ?

- Ce n'est plus la peine, je le connais d'avance.

Notons tout d'abord que l'IMP y renvoie systématiquement à une situation passée, mais toujours saillante - ou en tout cas non périmée - au moment de l'énonciation. Dans (17a), cette situation passée est directement visible dans les phrases qui précèdent l'énoncé à l'IMP : l'emploi de cette forme verbale permet à la pharmacienne d'embrayer avec la situation - passée, mais toujours saillante, car non (encore) satisfaite - où son jeune interlocuteur cherchait à se peser. Dans (17b), même si aucune situation passée valide en $t_{0} n^{\prime}$ est directement présente dans le co-texte, on ne prend pas de gros risques en affirmant qu'il suffirait de remonter à un contexte plus large pour la retrouver. En employant tw voulais, le locuteur fait clairement entendre qu'il avait déjà perçu des signes de cette volonté non encore (explicitement) exprimée, et, en l'occurrence, il ne s'est pas trompé. Cette explication peut s'appuyer ici sur l'analyse syntaxique des interrogatives employées dans (17). L'interrogation totale y est marquée par l'intonation, et l'ordre linéaire des mots reste celui d'une assertion. C'est qu'on ne peut avoir, dans ce contexte, un IMP de ce type dans une 
interrogative marquée par l'inversion du clitique sujet. Ce type d'interrogatives ne peut s'employer en effet que lorsque le locuteur n'a pas la moindre idée de la validité de la relation prédicative. Or il cherche à dire ici exactement le contraire, à savoir qu'il a perçu des signes de ce désir, et qu'il en demande, tout au plus, la simple confirmation. Et c'est là précisément où réside l'effet " politesse " enregistré dans ces énoncés, effet distinct, selon nous, des deux autres formes déjà identifiées, i.e. la neutralisation d'un acte de langage, et la justification prudente d'une situation susceptible de gêner l'interlocuteur. La politesse dont il est ici question se confond en effet avec ce qu'on appelle couramment prévenance : en employant l'IMP, le locuteur se montre attentionné à l'égard de son interlocuteur, en montrant qu'il a perçu des signes de son désir avant même qu'il ne soit explicitement exprimé.

Cette analyse est très proche de celle que Berthonneau \& Kleiber (1994) proposent pour l'IMP forain. Or, il se trouve que l'IMP des phrases (17) est en tout point comparable à l'IMP forain, avec lequel il partage, en plus des propriétés interprétatives, un certain nombre de propriétés formelles: non seulement l'IMP de (17) est employé à la $2^{\circ}$ personne, qui peut naturellement se rencontrer avec l'IMP forain (en concurrence avec la $3^{\circ}$ personne, considérée, il est vrai, comme canonique), mais en plus, comme lui, il se rencontre exclusivement dans des énoncés interrogatifs. Or, ainsi que nous le verrons ci-dessous, l'interrogation est incompatible avec l'expression d'une atténuation par l'IMP. D'un point de vue chronologique, enfin, il s'agit d'un authentique IMP temporel, qui naît dans le passé et se prolonge, en vertu de ses propriétés aspectuelles, dans le présent. Cela signifie notamment qu'il n'est pas employé «à la place " d'un présent...

Peut-on considérer, alors, l'IMP des énoncés (17) comme atténuatif?

Au lieu de parler d'IMP de politesse, nous considérons qu'il s'agit plutôt d'un cas de "politesse à l'imparfait ": l'énoncé à l'IMP ne constitue pas ici la version atténuée de celui au présent, qui serait l'énoncé "d'origine", mais se présente à nos yeux comme un énoncé non marqué ${ }^{5}$. Difficile donc dans ce cas de parler d'atténuation (d'un énoncé "neutre »).

En ce qui concerne la $3^{e}$ personne, si on fait abstraction des énoncés dans lesquels le on est employé "à la place " de nous, aucun exemple de nos corpus n'a un sens atténuatif. On note toutefois la possibilité d'une lecture atténuative avec la $3^{\circ}$ personne dans l'exemple suivant que nous empruntons à Schogt (1968, cité par Berthonneau \& Kleiber, 1994) :

(18) Il voulait vous demander s'il est possible de suivre une option d'italien en plus. Nous sommes d'accord avec Schogt, Berthonneau \& Kleiber pour dire que l'atténuation est conditionnée ici par l'existence d'un lien étroit entre le locuteur et le référent de il (dans l'exemple ci-dessus, respectivement un père et son fils). Si un tel lien n'existe pas, l'IMP acquiert une lecture strictement temporelle. Autrement dit, dans ce genre d'énoncés, si la $1^{\text {re }}$ personne semble être la personne canonique qui déclenche la lecture atténuative, elle peut, lorsque le contexte le permet, céder la place à la $3^{\circ}$ personne si cette dernière se présente comme un porte-parole, un représentant légitime, de la personne-origine de la demande.

5. Sans doute devrions-nous alors considérer que c'est l'emploi du PR qui produit un certain effet «impolitesse ». 
En bref, le choix de la $1^{\text {re }}$ personne ne correspond pas ici à une contrainte syntaxique, mais à une contrainte interprétative: l'IMP atténuatif semble exiger d'être construit avec un sujet représentant, directement ou, beaucoup plus rarement, indirectement, le locuteur.

\subsection{Classes sémantiques de l'infinitif gouverné par l'imparfait d'atténuation}

Si la structure atténuative typique, telle qu'elle se dégage des descriptions antérieures, est une phrase dont le verbe principal, vouloir/venir à l'IMP, domine un infinitif de type demander/dire, la question se pose de savoir s'il est possible d'obtenir un effet de sens analogue avec une infinitive contenant d'autres types de verbes. L'existence d'un énoncé comme (11) suffit à montrer que, lorsque le verbe opérateur est venir, la présence dans l'infinitive d'un verbe de type demander/dire ne constitue pas une condition nécessaire à l'émergence de l'effet atténuatif. En ce qui concerne vouloir, un examen quantitatif des exemples atténuatifs rencontrés dans nos corpus tend à montrer le contraire, ainsi que nous pouvons l'observer dans le tableau suivant:

Corpus FRANTEXT

\begin{tabular}{|l|c|c|l|c|}
\hline Forme verbale & Total & Atténuation & Verbe de l'infinitive & Nombre d'occurrences \\
\hline voulais & 1222 & 43 & dire & 24 \\
& & & demander & 09 \\
& & & savoir & 07 \\
& & & féliciter & 01 \\
& & & voir & 01 \\
& & & présenter & 01 \\
\hline
\end{tabular}

Corpus d'Orléans

\begin{tabular}{|l|c|c|l|c|}
\hline Forme verbale & Total & Atténuation & Verbe de l'infinitive & Nombre d'occurrences \\
\hline voulais & 57 & 03 & $\begin{array}{l}\text { demander } \\
\text { savoir }\end{array}$ & 02 \\
& & & 01 \\
\hline
\end{tabular}

On constate que la majorité des énoncés avec vouloir à l'IMP atténuatif ont dans la subordonnée infinitive un noyau verbal de type demander ou dire. Si cela semble a priori confirmer la plupart des descriptions habituelles qui conditionnent la lecture atténuative de vouloir par la présence dans la subordonnée d'un infinitif de ce type, un examen plus détaillé montre cependant que l'effet atténuatif dont il est ici question correspond uniquement à la neutralisation d'un acte de langage. Autrement dit, ce n'est pas l'atténuation dans son ensemble, mais l'effet " neutralisation d'un acte de langage ", c'est-à-dire l'effet atténuatif de type déperformatif - considéré par nous comme l'effet-type déclenché par vouloir - qui semble être conditionné par la présence dans la subordonnée infinitive d'un verbe dire/demander. Cette contrainte s'explique par le fait que si vouloir domine un infinitif d'un autre type, il ne peut avoir qu'une lecture temporelle du passé, qui, dans notre conception, exclut la lecture atténuative de type déperformatif. L'imparfait de vouloir dominant un infinitif d'un type autre que dire/demander peut alors avoir soit une lecture strictement 
temporelle, soit une lecture atténuative de type justificatif. Le premier cas se réalise lorsque l'IMP ne commute pas avec le présent, comme dans :

(20) Il entraîna Julien au laboratoire et referma la porte derrière eux. Un moment ils restèrent debout l'un en face de l'autre à se regarder, puis le chef dit doucement :

- Je voulais te voir seul avant de m'en aller.

Lorsque l'IMP peut être remplacé par un PR, on obtient des énoncés atténuatifs comme :

(21) Je m'en vais, je suis au bout du couloir et cette putain de porte s'ouvre. Le type a des lunettes et des cheveux raides et noirs. Je bredouille :

"J'voulais voir Jérôme..."

où non seulement l'IMP renvoie au passé, mais en plus, l'effet atténuatif n'est rendu que parce que l'IMP renvoie au passé : justifier une intrusion, un dérangement, plus généralement, expliquer une situation présente par une intention passée. On aura noté que, dans ce cas, vouloir commute avec venir.

En bref, l'IMP dominant une infinitive ne peut être atténuatif-déperformatif que si le noyau verbal de l'infinitive est d'un type particulier, contenant dire/ demander. Mais, dans ce cas, qu'en est-il de l'énoncé suivant, avec féliciter, répertorié dans le tableau (19)?

(22) Je m'appelle Pierre Gauthier. Je voulais vous féliciter pour ce que vous avez. dit tout à l'heure.

Si cet énoncé relève bien de l'atténuation déperformative ${ }^{6}$, quel est le point commun aux verbes dire, demander et féliciter? Pour nous, une réponse s'impose tout naturellement. Tous ces verbes ont finalement en commun d'appartenir à la catégorie des verbes performatifs (ou en emploi performatif) : si quelqu'un dit $l c$ dis $P$, il dit $P$; s'il dit $J e^{\prime}$ demande si $P$, il demande si $P$; s'il énonce $J c^{\prime}$ te fúlicite, il félicite l'interlocuteur.

L'avantage de cette analyse, si elle se confirme, est qu'elle peut s'étendre aux énoncés atténuatifs avec vouloir dominant une complétive, comme dans (7), où le sens atténuatif, on s'en souvient, est conditionné par la présence d'un acte de langage de type injonctif. Il reste tout de même, avant d'adopter définitivement cette analyse, un obstacle de taille: les énoncés atténuatifs en question ne se présentent pas sous la forme canonique des énoncés performatifs $l c$ dis/demande $P$, mais sous la forme Je voulais dire/demander $P$. Nous y reviendrons.

\subsection{Vouloir : verbe transparent ?}

Une première remarque descriptive s'impose ici : tous les énoncés considérés intuitivement par nous comme atténuatifs déperformatifs sont affirmatifs (non négatifs, non interrogatifs).

En effet, si vouloir est affecté par une négation, il ne peut en aucun cas exprimer une atténuation, y compris dans les contextes syntaxiques et sémantiques les plus

6. L'autre analyse, concurrente, ne nous semble pas absurde, qui reviendrait à expliquer l'effet "politesse " ici comme le résultat d'une justification de l'intrusion du locuteur dans la sphère d'un interlocuteur inconnu. 
favorables. C'est ce qui ressort, par exemple, de l'examen de l'énoncé non atténuatif suivant:

- Et ta mère?

- Maman est morte.

- Pardon! Je ne voulais pas te faire de peine.

où seule la lecture temporelle est disponible: l'IMP, qui ne peut pas être remplacé par un présent, y renvoie au passé (proche). Mais nous pouvons aller plus loin, en affirmant que ces énoncés négatifs, qui sont perçus comme " polis ", ne peuvent pas exprimer une atténuation, même débarrassés de tout le co-texte exprimant le passé, et quel que soit le contexte pragmatique imaginé.

Se pose maintenant la question de l'interrogation : vouloir peut-il se combiner avec une interrogation tout en continuant à exprimer une atténuation? Une première indication nous est fournie par l'examen des énoncés atténuatifs déperformatifs attestés : aucun d'entre eux n'est de type interrogatif. Parallèlement, dans nos corpus, aucun énoncé interrogatif n'exprime une atténuation déperformative. Cette observation ne doit rien aux aléas de corpus, puisque, dès qu'il est affecté par une interrogation, chacun de nos énoncés atténuatifs cesse d'exprimer toute atténuation.

En bref, pour qu'il soit atténuatif déperformatif, l'IMP du verbe vouloir doit être affirmatif.

Mise en rapport avec un certain nombre d'indices, cette constatation suggère une hypothèse, assez intuitive, qui reviendrait à rapprocher vouloir, dans ces énoncés, de la catégorie des verbes transparents.

Sans avoir toutes les caractéristiques syntaxiques du verbe transparent, vouloir à l'IMP atténuatif en est en effet sémantiquement très proche. D'abord, on vient de le voir, vouloir à l'IMP atténuatif, comme tout verbe transparent, n'est pas passible d'une négation ou d'une interrogation. Ensuite, non seulement ce verbe s'emploie exclusivement à la $1^{\text {re }}$ personne (ou à la $3^{e}$ lorsqu'elle se présente comme l'intercesseur de la $1^{\mathrm{r}}$ ), mais en plus il fait partie des emplois verbaux présentant une asymétrie systématique entre la $1^{\text {re }}$ et les autres personnes : conjugué à une autre personne, il cesse d'être atténuatif, comme les verbes transparents cessent d'être transparents lorsqu'ils sont conjugués à une personne autre que la $1^{\text {re }}$. Enfin, comme tout verbe transparent, l'IMP atténuatif localise le procès dans le Présent du locuteur ${ }^{7}$ : I'IMP cesse d'être atténuatif-déperformatif lorsqu'il renvoie au passé, tout comme le verbe transparent cesse d'être transparent dès qu'il renvoie à une époque autre que le PR.

Il nous reste tout de même à expliquer pourquoi nous considérons vouloir dans les énoncés atténuatifs comme un verbe transparent, alors qu'il ne semble pas en avoir la caractéristique principale. Un verbe est en effet généralement considéré comme transparent s'il gouverne une phrase de type que-P et que l'énoncé global

\footnotetext{
7. Contrairement aux descriptions habituelles, qui lient la notion de transparence au I'R, en tant que forme verbale, il nous paraît clair qu'il ne peut pas s'agir d'une contrainte grammaticale, mais d'une contrainte référentielle. Ce ne sont pas en effet les formes verbales qui excluent la transparence ou la rendent possible, mais la référence temporelle: un verbe au P'R historique, par exemple, ne peut pas être transparent; un verbe conjugué à un temps de l'indicatif autre que le P'R, mais renvoyant au l'résent peut l'être. Pour plus de détails, voir Abouda (1997).
} 
équivaut à peu près à l'énoncé de la phrase $P$ indépendante, sans le verbe introducteur. C'est, par exemple, le cas du verbe transparent croirc:

(a) Je crois que Pierre est un bon candidat

(b) I'ierre est un bon candidat.

Si, pour des raisons syntaxiques évidentes, ce test ne peut pas s'appliquer ici tel quel, il peut être facilement adapté : je voulais dire/demander sera considéré, à l'effet d'atténuation près, comme sémantiquement équivalent à je dis/demande.

L'hypothèse du traitement de vouloir comme verbe transparent semble se confirmer par l'examen de nos énoncés atténuatifs, dont une bonne part (13 sur 43) se présente non pas sous la forme complète Je voulais dirc/demander $P$, mais sous la forme incomplète $J c$ voulais dire/demander, interrompue par un locuteur hésitant, à la recherche de ses mots:

(25) (a) Pendant qu'il remplissait les papiers, j'ai toussé délicatement dans mon poing :

- Ah, docteur, je voulais vous dire... Il y a aussi une petite chose qui me tracasse...

(b) ... à ce moment s'éleva la voix du chevalier. Elle était basse et méditative, comme s'il se parlait à lui-même.

- Je voulais vous dire, néanmoins...

Or, tous ces énoncés atténuatifs incomplets se présentent systématiquement sous la forme (26a), jamais sous la forme (26b) :

(26) (a) Je voulais te dire... /Je voulais te demander...

(b) Je voulais... Je voulais te dire/demander

Cela tend à montrer que la suite voulais + dirc/demander ne se présente pas comme l'addition d'un verbe vouloir et d'un verbe dire/demander, mais comme un verbe dire/demander dans une version atténuée. Notre hypothèse revient ainsi à dire que cette suite fonctionne comme une même et unique forme verbale, formée d'une sorte d'auxiliaire modal suivi d'un infinitif, qui se charge de véhiculer la vraie valeur sémantique du verbe.

Cette hypothèse permet d'abord de comprendre pourquoi, contrairement aux traitements habituels, nous considérons l'infinitif des énoncés atténuatifs déperformatifs comme un verbe performatif : c'est parce que le verbe vouloir est transparent que la suite fe voulais dire/demander se présente comme sémantiquement équivalente à Je dis/demande. Autrement dit, la performativité de l'infinitif est rendue possible par la transparence du verbe "opérateur".

Cette hypothèse permet également de comprendre pourquoi souhaiter et désirer ne permettent pas, malgré toute leur proximité sémantique, de donner naissance à un IMP atténuatif : parce qu'il est verbe transparent, vouloir paraît nettement moins marqué que souhaiter ou désirer, aussi bien sur le plan stylistique que sur le plan sémantique. C'est cette même transparence qui fait de vouloir le seul des trois verbes considérés à pouvoir s'employer dans des phrases impératives dans lesquelles il domine un infinitif : Veuillez partir (comme équivalent atténué de Partez!) vs *Désirez partir ${ }^{*}$ Souhaitez partir" . Cette hypothèse semble être elle-même confirmée par l'impossibilité de former une phrase impérative avec vouloir gouvernant un SN: *Veuillez un gâteau. C'est qu'il s'agit en l'occurrence d'un

8. Voir à ce propos D. Leeman (1994). 
verbe plein, qui se comporte comme les deux autres verbes désidératifs, et ne permet donc pas la formation de phrases impératives. Tout cela revient à admettre que vouloir se présente comme un simple auxiliaire modal aussi bien dans l'atténuation à l'imparfait que dans l'atténuation injonctive...

L'analyse de vouloir comme verbe transparent dans les énoncés atténuatifs permet ainsi de comprendre pourquoi nous considérons que $J c$ voulais dire/ demander n'est pas l'atténuation de Je veux dire/demander, mais comme l'atténuation de Je dis/demande. Autrement dit, il s'agit pour nous de l'atténuation d'un acte de langage, et non pas celle d'une volonté.

\section{VOULOIR ET VENIR, REPRÉSENTANTS DE DEUX TYPES D'EMPLOI ATTÉNUATIF}

Un examen détaillé des conditions syntaxiques et discursives des énoncés dits «atténuatifs " nous a mis sur la piste de l'existence de deux types d'énoncés, et partant de deux types d'emplois pour l'IMP d'atténuation, i.e. un IMP déperformatif et un IMP justificatif. Même si les deux types ne s'opposent pas diamétralement (certains énoncés, nous l'avons vu, sont passibles des deux analyses), et ont même en commun un certain nombre de traits qui justifient qu'on les regroupe au sein d'énoncés " atténuatifs ", ils n'ont pas exactement les mêmes traits syntaxiques, pas plus que les mêmes fonctions discursives. Nous nous proposons de passer en revue les principales différences syntaxiques, chronologiques, et sémantiques qui opposent ces deux types d'énoncés.

Sur le plan syntaxique, d'abord, rappelons que les énoncés atténuatifs déperformatifs ont pour structure typique, voire quasi exclusive, vouloir à l'IMP et à la 1 re personne dominant une infinitive dont le noyau verbal est de type dire/ demander. Le verbe vouloir, nous venons de le voir, a ici toutes les propriétés du verbe transparent, ce qui rend possible de le traiter comme simple support du morphème de l'IMP. Ce trait permet de traiter ces phrases à $V 1 I M P+V 2 I N F$ comme équivalentes, à l'effet d'atténuation près, à $V 2$ au PR (Je voulais vous dire $p=$ $J e$ vous dis $p . .$.$) . Cette propriété permet ainsi de comprendre pourquoi vouloir peut$ naturellement être employé dans ce type d'énoncés, alors que des verbes comme souhaiter ou désirer, malgré leur proximité sémantique, ne le peuvent pas.

Les énoncés à IMP justificatif s'opposent syntaxiquement aux énoncés atténuatifs sur ces deux points. D'abord, ils se rencontrent dans des contextes syntaxiques beaucoup plus libres, et avec des verbes assez variés, dont notamment venir, passer, chercher, téléphoner, etc., mais aussi vouloir. D'autre part, contrairement aux énoncés déperformatifs, les énoncés justificatifs ont pour noyau des verbes pleins. Lorsqu'il y a un verbe infinitif gouverné par l'IMP, la structure V1IMP +V2INF $\mathrm{n}^{\prime}$ est donc jamais paraphrasable par $V 2 P R$ (Je venais passer l'aspirateur $\neq$ le passe l'aspirateur; Nous cherchions à joindre $X \neq$ Nous joignons $X$, etc.)...

Sur le plan chronologique, deux questions se posent: (i) à quelle portion du temps chronologique renvoie l'IMP? (ii) par quel mécanisme arrive-t-il à assurer la validité du procès dans le présent du locuteur? Sur ces deux points, les réponses diffèrent selon que l'on a un IMP déperformatif ou un IMP justificatif.

L'IMI déperformatif peut, plus ou moins naturellement, être remplacé par un conditionnel, mais cette substitution ne s'accompagne pas d'un changement chronologique. Cela montre selon nous que le verbe vouloir, dans ce type d'emploi, reste 
imperméable à toute chronologie. Nous rapprochons cette propriété, assez singulière, de son statut de verbe transparent. D'autre part, si le remplacement d'un IMP déperformatif par un présent ne pose théoriquement aucun problème (1'IMP y est employé à la place du PR), nous pouvons légitimement nous interroger sur le naturel d'énoncés de type Je veux te dire que $P$ ou Je veux vous demander $P$.

Le remplacement de cet IMP par un conditionnel ou par un PR confirme donc l'essentiel de nos analyses avancées ci-dessus, selon lesquelles vouloir dans cet emploi se comporte comme simple support du morphème de l'IMP, morphème dépourvu d'ailleurs en l'occurrence de toute chronologie, et qui se charge simplement de véhiculer l'effet d'atténuation.

Par quel mécanisme l'actualité de ces énoncés est-elle assurée ? La réponse nous semble claire : c'est la performativité qui se charge ici de signifier l'actualité. La lecture par défaut de Je voulais te dire $P$ est bien la lecture atténuative, qui va de pair avec la lecture performative. L'énoncé se présente alors comme $P$, dit poliment.

Sur ces deux points, l'IMP justificatif est sensiblement différent. D'abord, il est clairement porteur d'une valeur chronologique : son remplacement par un PR permet de le situer dans le Présent. Cela présuppose que cet IMP situe le procès dans le passé du locuteur, ce qui ne contredit pas l'analyse défendue ci-dessus selon laquelle cet emploi présente les raisons de la venue du locuteur quand elles étaient au stade de projet, d'intentions, en $t_{(0-n}$. Sauf que parler d'une situation en $t_{(0)-n}$ ne signifie pas forcément que la situation cesse d'être valide en $t_{0}$. Une indication quelconque peut suffire à signifier que le procès reste toujours d'actualité. En l'occurrence, cette actualité est rendue par un marquage pragmatique : je raconte les objectifs de ma venue, objectifs dont on sait qu'ils ne sont pas réalisés, et qu'ils sont donc toujours d'actualité. En bref, l'IMP justificatif garde sa valeur de temps passé, puisque, en l'occurrence, c'est cet aspect passé qui permet d'exprimer l'atténuation.

Enfin, sur le plan discursif, l'énoncé déperformatif se présente comme l'atténuation ou la neutralisation d'un acte de langage (le plus souvent assertif ou interrogatif), tandis que l'énoncé justificatif prend la forme d'une justification de l'intrusion du locuteur dans la sphère de son interlocuteur. Dans le premier cas, l'aspect performatif assure à l'énoncé un aspect offensif : en disant Je voulais te dire $q u e ~ P$, je dis $P$, et il $n^{\prime} y$ a plus aucun moyen de changer d'avis. Il y a ici un mélange d'atténuation et de fait accompli, voire un mélange de politesse et de renforcement. Avec l'IMP de justification, au contraire, aucun acte de langage n'est exprimé, aucune demande n'est non plus - explicitement - exprimée et le locuteur, loin de se présenter comme sujet de l'énonciation, se présente alors beaucoup plus sur la défensive, comme simple sujet - objet ? - de l'énoncé...

\section{EN GUISE DE CONCLUSION}

Cet article avait pour objectif d'étudier l'imparfait d'atténuation en déterminant ses propriétés saillantes, à la fois externes - notamment ses environnements syntaxiques - et internes - principalement, ses caractéristiques chronologiques et le mécanisme précis lui permettant de produire un effet d'atténuation. L'examen de ces différentes propriétés nous a permis d'établir l'existence de deux types d'IMP atténuatif, un IMP justificatif et un IMP déperformatif.

Si, pour le premier, ce sont finalement ses propriétés temporelles et aspectuelles habituelles qui lui permettent de produire un effet d'atténuation, se pose la 
question de savoir par quel mécanisme exact le morphème IMP rend une atténuation de type déperformatif. L'atténuation déperformative, nous l'avons vu, correspond à la neutralisation d'un acte de langage, elle-même largement assurée par l'aspect indirect de la demande. Mais pourquoi est-ce un imparfait ? Après tout, si c'est l'aspect indirect qui constitue le facteur décisif permettant la production d'une atténuation déperformative, on s'attend a priori à ce que n'importe quelle autre forme temporelle puisse en faire de même. Or ce n'est pas le cas. Si on fait ici abstraction du conditionnel ${ }^{9}$, aucune forme temporelle de l'indicatif autre que l'IMP n'est en effet en mesure de produire une atténuation déperformative. Contrairement à un certain nombre de descriptions antérieures qui, au prix quelquefois de quelques acrobaties, voire d'explications contre-intuitives, s'évertuent à expliquer le phénomène par les propriétés aspectuelles et temporelles de l'imparfait, considéré comme un temps du passé, nous pensons que l’IMP déperformatif est dépourvu de toute chronologie. Nous considérons en effet, suivant en cela une longue lignée de travaux dont les représentants les plus illustres sont Damourette \& P'ichon, que l'lMP est un simple non-PR, pouvant s'opposer à lui, certes sur le plan chronologique, mais aussi sur beaucoup d'autres plans, et notamment sur le plan énonciatif. Du coup, si - ainsi que l'écrivent Damourette \& Pichon ( $\$ 1740$ : 226) - le propre de l'IMP dans tous ses emplois est « l'abandon du repérage par rapport au moi-ici-maintenant ", nous comprenons pourquoi c'est le morphème IMP qui s'agglutine au verbe transparent vouloir afin de neutraliser un acte de langage : l'IMP, exprimant par définition une sorte de NON-[Moi-Ici-Maintenant], est bien placé pour introduire la modalisation qui consiste à altérer la prise en charge de l'énoncé, à l'attribuer à ce non-Moi, à cet autre Moi, au locuteur en tant qu'être du monde, par opposition au locuteur en tant que tel ${ }^{10}$...

\section{Bibliographie}

ABOUDA. L. (1997). Recherches sur la syntaxe et la sémantuque du conditionnel en fronçars mocjerne, Thèse. Université Paris 7

ABOUDA. L. (2001). « Les emplois joumalistique, polémique, et atténuatif du conditionnel. Un traitement unitaire », in P. Dendale \& L. Tasmowski (éds.). Le conditionnel en fronçois, Metz, Université de Metz/ Paris, Kincksieck. Collection Recherches Linguisticiues, $n^{\circ}$ 25, 277-294.

BerihONNEAU. A.M. \& KLEBBE.R. G. (1993). "Pour une nouvelle approche de l'imparfait. L'imparfait, un temps anaphorique méronomique $\gg$, Longoges, $112,55-72$

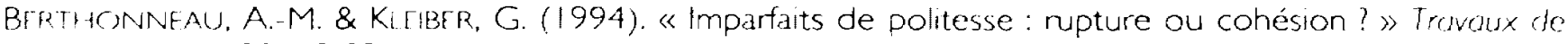
Imgustique, $29.59-92$.

DAMOUREITE, I. \& PICHON. E. (1911-1940). Des mots a lo pensée : essoi de grammare de la longue fresncruse. Paris, D'Artrey, 1970.

DUMROR. O. (1984). Le dire et le dit. Paris, Minuit

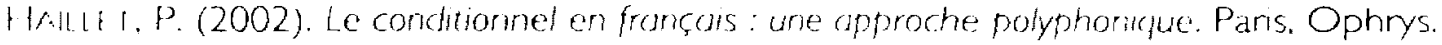

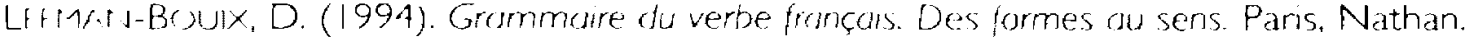

Sc.16x,1, 1.G. (1968). Le système verbos du fruncus contemporcin. La Hayc. Paris, Mouton.

Kuleallk. C. (1996). Le systeme verbol fromcers. Paris, Amand Colin.

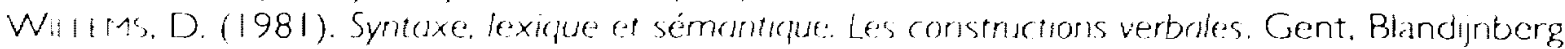

Wil14 1. M. (1996). "L.imparfait: le temps des anaphores ». Cohers Chronos, 1. 199-215.

4. Voir Abouda (2001).

10. Voir I Jucrot (1984). 